\title{
Two Contrasting African Easterly Wave Behaviors
}

\author{
YuAN-Ming CHENG AND CHRIS D. THORNCROFT \\ Department of Atmospheric and Environmental Sciences, University at Albany, State University \\ of New York, Albany, New York \\ GEORGE N. KILADIS \\ Physical Sciences Division, NOAA/Earth System Research Laboratory, Boulder, Colorado
}

(Manuscript received 4 October 2018, in final form 16 February 2019)

\begin{abstract}
The dominant structural variability of African easterly waves (AEWs) is explored using an empirical orthogonal function (EOF) approach. The structure of AEWs is obtained by projecting the wind fields from reanalysis data and satellite-derived brightness temperature $T_{b}$ onto the principal components associated with EOF patterns of filtered $T_{b}\left(T_{b} \mathrm{EOF}\right)$ and $700-\mathrm{hPa}$ meridional wind (v700 EOF). The wave structure depicted by the $T_{b}$ EOF has confined convection and circulation mostly south of the African easterly jet. It shares many characteristics with AEWs analyzed and discussed in the literature. In contrast, the v700 EOF exhibits less familiar characteristics and includes interactions with the equatorial and subtropical regions. The convective patterns are characterized by a "checkerboard" pattern of convection that has not been emphasized before. The most striking feature is the broad meridional extent, which depicts interactions with a mixed Rossby-gravity wave (MRG) in the equatorial region and interactions with the basic-state potential vorticity in the subtropics. The southern portion of the wave has a modified MRG structure, and this AEW-MRG hybrid cannot be separated using the EOF technique, indicating the prevalence of such structures. The subtropical interaction at mid- to lower levels establishes a vortex off the coast of Morocco that results in dry-air advection into the tropics in tandem with the northern vortex. At upper levels, a subtropical wave train is induced by the AEW-associated convective inflow and outflow. The contrasting AEW circulations are associated with differences in the precipitation rates and patterns over Africa. These results highlight the variability of AEW structures and their interactions with equatorial and subtropical waves.
\end{abstract}

\section{Introduction}

Our perception of African easterly waves (AEWs) has been consolidated through several key composite studies (e.g., Carlson 1969; Burpee 1975; Reed et al. 1977; Duvel 1990; Kiladis et al. 2006, hereafter KTH2006). While these mean structures and statistics are useful and much conceptual and dynamical understanding has been gained from them, evidence has shown that AEWs can vary substantially in terms of their structure and amplitude (e.g., Berry et al. 2007; Zawislak and Zipser 2010; Bain et al. 2011), their activity levels (e.g., Leroux and Hall 2009), as well as their genesis location and tracks (e.g., Mekonnen et al. 2006; Berry et al. 2007). Understanding such variability has economic and societal implications as AEWs are the primary rain producers in the

Corresponding author: Yuan-Ming Cheng, ycheng4@albany.edu semiarid Sahel and can influence the probability of tropical cyclone genesis in the tropical Atlantic. However, few have attempted to address such variability and its causes, and our knowledge is limited in this area. As a first step to more thoroughly documenting the variability of AEWs, this paper explores the nature of AEW structural variability.

AEWs are recognized as the dominant weather systems over tropical Africa and Atlantic in boreal summer. The large-scale setup of the West African monsoon allows these systems to amplify through distinct Rossby wave dynamics and to exhibit structural characteristics unique to the region (Lau and Lau 1990; Thorncroft and Hoskins 1994). On average, these transient, synopticscale disturbances have periods of 3-5 days with wavelengths of $2500-4000 \mathrm{~km}$ and propagate westward at about 8-12 $\mathrm{m} \mathrm{s}^{-1}$ (e.g., Carlson 1969; Reed et al. 1977; Diedhiou et al. 1999; KTH2006). Observational studies 
have shown that the mean AEW structure has a peak amplitude in dynamical fields occurring close to the level of the African easterly jet (AEJ) in the rainy belt equatorward of the AEJ as well as near the surface baroclinic zone at the southern fringe of the Saharan heat low. In the literature, the structure associated with the former is often referred to as the southern wave and the latter the northern vortex (NV). The observed trough axes of the southern wave are tilted from southwest to northeast equatorward of the AEJ and from southeast to northwest poleward of the AEJ axis, suggesting barotropic wave growth. In the low-level baroclinic zone around $20^{\circ} \mathrm{N}$, the circulation slopes eastward with height in the mid- to lower levels, indicative of baroclinic energy conversions (e.g., Reed et al. 1977; Norquist et al. 1977; KTH2006; Hsieh and Cook 2007). Composite studies have also established the relationship between AEWs and convection (Reed et al. 1977; Duvel 1990; Fink and Reiner 2003; KTH2006; Janiga and Thorncroft 2013). Equatorward of the AEJ, the most intense convection is generally observed ahead of the trough over land and within the trough over the ocean, while poleward of the jet, it tends to be located behind the trough (Duvel 1990; Fink and Reiner 2003; KTH2006). These studies outline the mean structure of AEWs and provide a framework to understand AEW dynamics.

It is a natural development to use our knowledge of the "typical" AEW structure to assess how it varies. In a case study of an AEW, Bain et al. (2011) concluded that the complicated potential vorticity $(\mathrm{PV})$ structure of their wave did not conform to the known AEW archetype. Therefore, it was advocated that a more comprehensive model for AEW structures should be included. By tracking waves over a season, Berry et al. (2007) found that, while the majority of mature AEWs exhibit meridionally broad cross-AEJ structures with a coherent NV, 20\% had only an isolated PV maximum confined equatorward of the AEJ with no discernable NV. These studies provide perspectives on the structures of AEWs and highlight variability in AEW behaviors. Nonetheless, a more thorough survey on the variability of $\mathrm{AEW}$ structures and its causes is still lacking.

To address the AEW structural variability, we use a statistical approach to isolate the dominant synoptic transient features in the West African monsoon during the boreal summer. This paper is organized as follows: Section 2 introduces the data and methodology. A brief introduction of the climatological basic state of the West African monsoon is given in section 3. Sections 4 and 5 encompass the primary results for AEW structures. Summary and discussion are presented in section 6 .

\section{Data and methods}

\section{a. Datasets}

In this study, the brightness temperature $T_{b}$ from the Cloud Archive User Services dataset (Hodges et al. 2000) during July-September from 1984 to 2013 was employed as a proxy for convection. The reanalysis data from ERA-Interim (Dee et al. 2011) for the same period were also used. The ERA dataset has been shown to be capable of representing AEWs (e.g., Reed et al. 1988; Diedhiou et al. 1999; KTH2006; Janiga and Thorncroft 2013) and realistically capture their vertical structure (KTH2006). Janiga and Thorncroft (2013) concluded that the differences in AEW structure among reanalysis datasets are related to the differences in precipitation and diabatic heating and that ERAInterim shows the least biases of precipitation over Africa compared with other reanalyses. These previous studies support the use of ERA-Interim dataset to study AEW structure. The four synoptic times in the 30 -yr period were chosen to match the availability from each dataset. To optimize between computational efficiency and ability to resolve the synoptic waves, both datasets were regridded onto a horizontal resolution of $2.5^{\circ}$. To estimate the rainfall, the $3 \mathrm{~B} 42$ product from the Tropical Rainfall Measuring Mission (TRMM; Huffman et al. 2007) was used. The data available from 1998 to 2013 at synoptic hours of the reanalysis were incorporated. The grid was kept at the original $0.25^{\circ}$ to give a full-detailed pattern of precipitation.

\section{b. Empirical orthogonal functions}

An empirical orthogonal function (EOF) analysis was used in this study. This method has been extensively applied to study wave structures in the tropics (e.g., Lau and Lau 1990; Kiladis et al. 2016). By maximizing covariance across the whole domain, the calculation of EOFs emphasizes the coherent and dominant patterns that occur simultaneously. The variables used in the EOF analysis were filtered and area weighted before calculating the eigenvectors of the covariance matrix. The associated structure of the EOF pattern is then attained by regressing the raw fields of interest onto the principal components (PCs) of the EOFs. The wave structure is obtained by scaling the PC to one standard deviation, corresponding to a moderate wave. By calculating regression at lags, the evolution of the wave structure can also be studied. The statistical significance between the predictand and predictor is determined by the correlation with a standard $t$ test after the degrees of freedom were estimated using autocorrelation characteristics of both time series (Livezey and Chen 1983). 
In addition to the anomalous fields in the regression method, rainfall composites were made to reflect total fields. The AEW amplitude and phase can be defined as (e.g., Lau and Lau 1992; Rydbeck and Maloney 2014)

$$
\begin{aligned}
\text { Amplitude } & =\sqrt{\mathrm{PC}^{2}+\mathrm{PC}^{2}}, \\
\text { Phase } & =\tan ^{-1}\left(\frac{\mathrm{PC} 2}{\mathrm{PC} 1}\right) .
\end{aligned}
$$

The phase can be further divided into eight phases, each with $45^{\circ}$, from $0^{\circ}$ to $360^{\circ}$. The AEW events were selected if the amplitude of the normalized PC pair was over 0.75 with eight consecutive phases.

Two variables, $T_{b}$ and 700-hPa meridional wind, were chosen to conduct two separate EOF analyses $\left(T_{b} \mathrm{EOF}\right.$ and v700 EOF, respectively) to extract the dominant structures of AEWs. Both variables have been commonly used as indices to study AEWs (e.g., Reed et al. 1977; Pytharoulis and Thorncroft 1999; KTH2006; Mekonnen et al. 2006). To focus on the AEW-associated convection following KTH2006, $T_{b}$ is filtered to retain the 2-6-day westward power before applying the EOF analysis. Note that the filter applied here is slightly different from the tropical depression (TD) band used in KTH2006 (see their Fig. 1), which includes the westward symmetric power with zonal wavenumbers of 6-20 and periods from 2 to (estimated) 7 days. To account for the asymmetric AEW signal about the equator, westward signals with wavenumbers $6-20$ and periods between 2 and 6 days in the Northern Hemisphere were included in the current study. For $700-\mathrm{hPa}$ meridional wind, a Lanczos filter (Duchon 1979) with 61 weights was applied to focus on the 2-6-day transient wave signals (Mekonnen et al. 2006; Leroux et al. 2010).

Analysis of EOFs based on $T_{b}$ and $700 \mathrm{hPa}$-meridional wind were repeated for different domains and domain sizes encompassed within $60^{\circ} \mathrm{W}-50^{\circ} \mathrm{E}$ and $35^{\circ} \mathrm{N}-30^{\circ} \mathrm{S}$ ( $30^{\circ} \mathrm{N}-30^{\circ} \mathrm{S}$ for $T_{b}$ EOF because of data coverage). Results presented in this paper were not found to be sensitive to the different domains. We also explored the sensitivity of our results with related variables including TRMM precipitation and 700-hPa vorticity (including curvature vorticity). The EOFs for the TRMM precipitation and 700-hPa vorticity are comparable to the $T_{b}$ EOF and v700 EOF, respectively. Finally, the sensitivity to the applied filters was also explored. The primary sensitivity found was with respect to the space filter on $T_{b}$ and time filter on the 700-hPa meridional wind. Excluding the westward constraint on $T_{b}$ gave a stronger signal over the Ethiopian highlands and Congo basin at the expense of AEW structures. Broadening the range to $2-10$ days in meridional wind resulted in greater emphasis on subtropical structures. Given our interest in AEW structures, we limited the time filter to 2-6 days on both $T_{b}$ and $700-\mathrm{hPa}$ meridional wind and applied an additional westward constraint on $T_{b}$. These filters are consistent with many previous studies and also consistent with prior reported spectra (e.g., KTH2006; Mekonnen et al. 2006). In addition, the AEW structures identified in this study were reproducible using a composite technique. Based on these analyses we conclude that the results presented in this paper are robust.

\section{Climatological basic state}

The large-scale environment in which AEWs develop is presented in Fig. 1. The West African monsoon is characterized by deep convection associated with the ITCZ, a midlevel easterly jet, and the associated PV strip. Figure 1a shows the climatological $T_{b}$ over JulySeptember from 1984 to 2013 and the standard deviation of 2-6-day westward-filtered $T_{b}$. The deepest convection lies zonally around $10^{\circ} \mathrm{N}$ with local maxima mostly collocated with topographical features such as the Guinea highlands, Cameroon highlands, and Darfur mountains in West, Central, and East Africa. The 2-6-day band accounts for more than $60 \%$ of the convective variance with periods greater than 2 days (Mekonnen et al. 2006). The 2-6-day westward activity is consistent across much of Africa except for east of $30^{\circ} \mathrm{E}$ where the activity rapidly declines despite a local convective maximum in the Ethiopian highlands. This feature indicates an increasing importance of synoptic-scale convective activity over West Africa as compared to East Africa. This is consistent with Mekonnen et al. (2006), where they show weaker AEW activity over East Africa compared to West Africa. The peak wave activity lies around $12.5^{\circ} \mathrm{N}$ over West Africa and moves to around $7.5^{\circ} \mathrm{N}$ over the Atlantic, becoming more collocated with the position of the timeaveraged maximum convection. As will be shown in section 4 , this suggests that the synoptic-scale waves tend to couple with convection poleward of the axis of mean peak convection over land while they weaken or reinforce convection along that axis offshore (KTH2006).

The climatological midlevel jet, commonly known as the AEJ, extends east-west across northern tropical Africa and the Atlantic Ocean with the maxima located at the west coast of the continent, peaking at around $15 \mathrm{~m} \mathrm{~s}^{-1}$ (Fig. 1b). As shown by both observational and numerical studies (e.g., Reed et al. 1977; Thorncroft and Hoskins 1994), the mean PV structure associated with the jet (Fig. 1c) and low-level baroclinicity satisfies the necessary conditions for baroclinic and barotropic growth, which is thought to contribute to the development of AEWs. Figure $1 b$ also overlays the standard deviation 

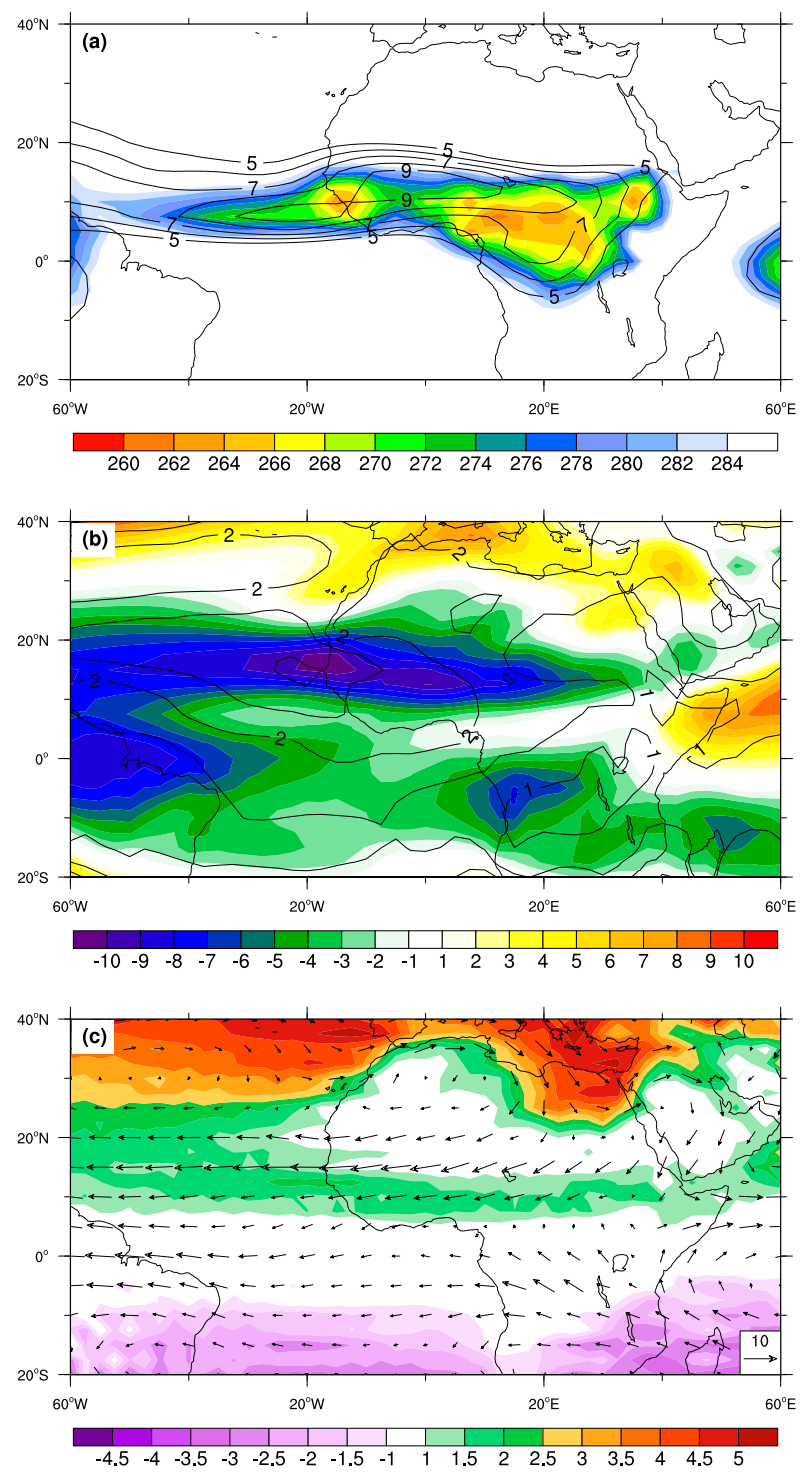

FIG. 1. July-September 1984-2013 climatological (a) $T_{b}$ (K; colors) and standard deviation of 2-6-day westward-filtered $T_{b}$ (K; contours), (b) 700-hPa zonal wind ( $\mathrm{m} \mathrm{s}^{-1}$; colors) and standard deviation of $2-$ 6-day-filtered $700-\mathrm{hPa}$ meridional wind ( $\mathrm{m} \mathrm{s}^{-1}$; contours), and (c) 700-hPa PV (10 $0^{-1} \mathrm{PVU} ; 1 \mathrm{PVU}=10^{-6} \mathrm{~m}^{2} \mathrm{~s}^{-1} \mathrm{~K} \mathrm{~kg}^{-1}$; colors) and wind vectors. In (a), the $T_{b}$ contours start at $5 \mathrm{~K}$ and has intervals of $1 \mathrm{~K}$. In (b), the zonal wind contour intervals are $0.5 \mathrm{~m} \mathrm{~s}^{-1}$ with the zero line omitted. In (c), the reference magnitude of $10 \mathrm{~m} \mathrm{~s}^{-1}$ for wind vectors is labeled in the bottom-right corner.

of 2-6-day of 700-hPa meridional wind. A zonally oriented but meridionally broad area of 2-6-day wave activity originates from tropical East Africa and extends westward to tropical Atlantic as well as southwestward into the Gulf of Guinea. The peak activity of the synoptic waves is located just south of the jet core and offshore of West Africa around $15^{\circ} \mathrm{N}$. Similar to Mekonnen et al. (2006) and the 2-6-day westward convective activity, there is an increase of synoptic activity from East to West Africa, but the activity defined by the meridional winds shows a broad meridional extent and weak gradient. This suggests that while the dynamical influence of the synoptic waves is widespread, the convective impact is more geographically constrained because of the more spatially limited favorable environment for convection (e.g., Thorncroft et al. 2011).

\section{Horizontal structure of $T_{b} \mathrm{EOF}$}

Figures $2 \mathrm{a}$ and $2 \mathrm{~b}$ show the first and second EOFs of the $T_{b}$ EOF. The first pair of EOFs together explains about $16 \%$ of the total variance, and they are separated from the rest of EOFs based on the criteria of North et al. (1982). The associated PCs have a maximum correlation coefficient of 0.93 with $\mathrm{PC} 1$ leading at 0.75 -day lag. Accordingly, the quadrature EOF pair together represents a westward-propagating wave signal with a period of about 3 days. Both EOFs depict alternating enhanced and suppressed convective anomalies from East Africa across the continent into the Atlantic. The most intense convective anomalies lie between $10^{\circ}$ and $15^{\circ} \mathrm{N}$ over land and shift to $5^{\circ}-10^{\circ} \mathrm{N}$ over the ocean, consistent with the observed maximum synoptic westward activity moving from around $12.5^{\circ}$ to $7.5^{\circ} \mathrm{N}$ (Fig. 1a).

The wave characteristics and circulation structure captured by the $T_{b}$ EOF can be obtained by regression with wind fields (Fig. 3). The enhanced (suppressed) convection is coupled with cyclonic (anticyclonic) circulation with wave axes tilting against the horizontal shear (e.g., Fig. 3c), a typical characteristic of observed AEWs. At $10^{\circ} \mathrm{N}$, an average wavelength of $3300 \mathrm{~km}$ is determined from the meridional wind at the simultaneous lag (Fig. 3c), falling in the range between 2100 and $4500 \mathrm{~km}$ reported in the previous AEW studies (Carlson 1969; Reed et al. 1977; Diedhiou et al. 1999; KTH2006). For phase speed, as documented in the literature (e.g., Carlson 1969; KTH2006), a slowdown of wave propagation from land to ocean is observed regardless of the variables used. The convective signal propagates westward at an average speed of $12.7 \mathrm{~m} \mathrm{~s}^{-1}$ over land and $7.9 \mathrm{~m} \mathrm{~s}^{-1}$ over the ocean. Tracking meridional wind gives an average phase speed of 13.6 and $9.1 \mathrm{~m} \mathrm{~s}^{-1}$, respectively. These statistics compare well with the AEW literature.

While the EOF patterns themselves do not have to correspond to any physical modes, physical interpretations can be gained using dynamical, kinematic, or statistical arguments (Monahan et al. 2009). The wave structure described in the previous paragraph, including the wave tilt and coupling with convection, and the consistent statistics with the AEW literature suggest the $T_{b}$ EOF captures the main features of observed AEWs. 

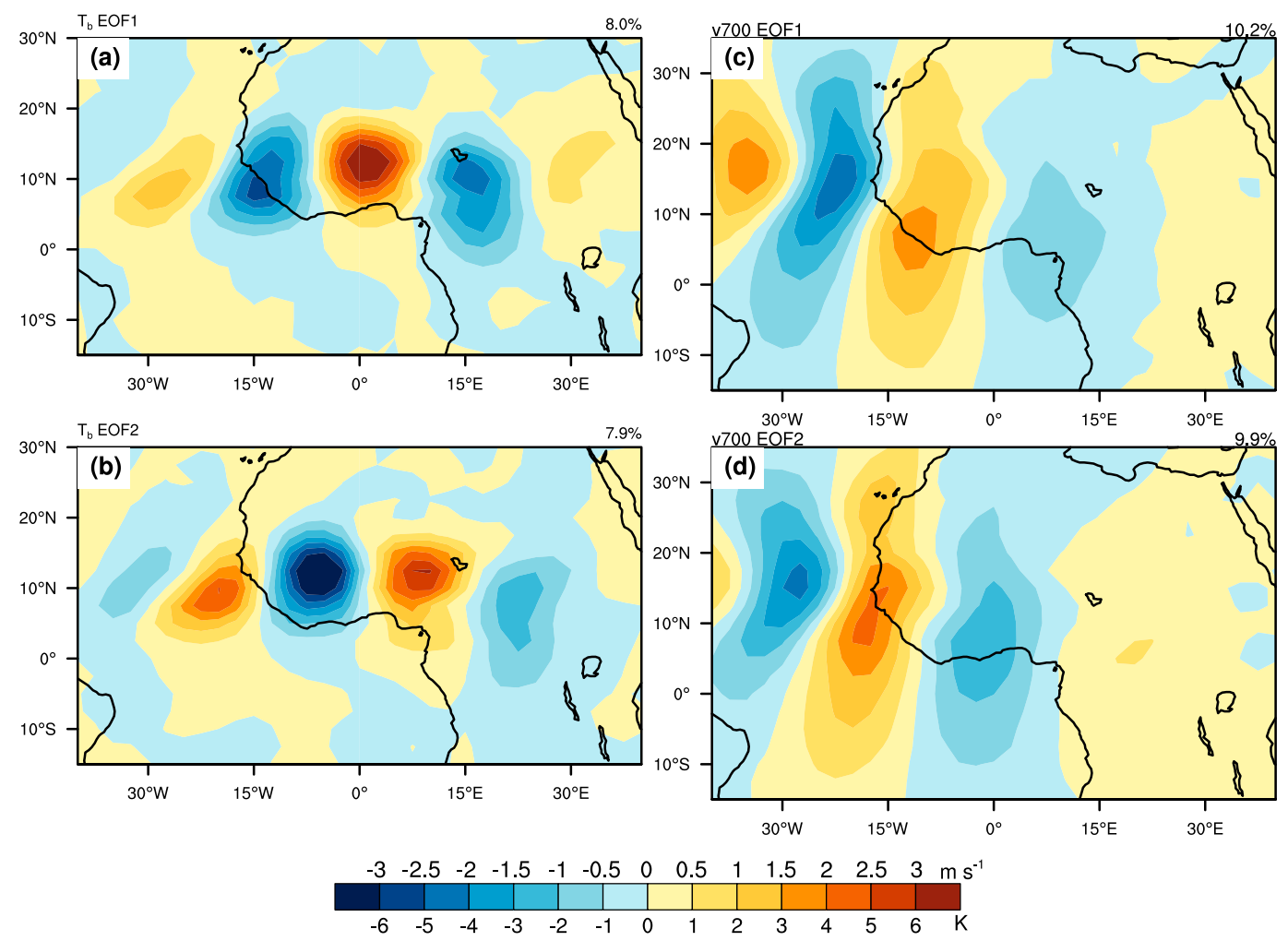

FIG. 2. (top) EOF1 and (bottom) EOF2 for (a),(b) 2-6-day westward-filtered $T_{b}$ (K) and (c),(d) 2-6-day-filtered meridional wind at $700 \mathrm{hPa}\left(\mathrm{m} \mathrm{s}^{-1}\right)$. The amplitude is scaled to one standard deviation of each corresponding PC. The variance explained by each EOF is indicated on the upper-right corner of each panel. Note the northern boundaries of the two kinds of EOFs differ because of data coverage.

The lag regression allows us to study the evolution of the wave structure (Fig. 3). The first signal of enhanced convection at the west coast on day 0 (Fig. 3c) appears as a broad convective area around $5^{\circ}-15^{\circ} \mathrm{N}, 20^{\circ}-35^{\circ} \mathrm{E}$ with weak circulation on day -5 (not shown). The topographically enhanced diurnal convection in those mountainous regions has been hypothesized to be critical in producing organized mesoscale convective systems that can initiate AEW development (e.g., Berry and Thorncroft 2005; Mekonnen et al. 2006; Thorncroft et al. 2008). Other studies have proposed that the waves are initiated by a preexisting AEW through upstream development (e.g., Diaz and Aiyyer 2013a,b). Those broad early convective signals can be interpreted as statistical evidence of AEW initiation. By day -3 (Fig. 3a), a clear convective signal is located around $10^{\circ} \mathrm{N}, 18^{\circ} \mathrm{E}$, and a weak cyclonic circulation has developed east of the convective center. The anomalous enhanced convection is mostly located ahead of the trough. By day -1.5 (Fig. 3b), the disturbance is found around the Greenwich meridian with a distinct circulation tilted from southwest to northeast, indicative of barotropic wave growth. The strongest convective enhancement occurs ahead of the trough in the northerlies and northeasterlies. By day 0 (Fig. 3c), the trough approaches the coast, and the location of the most intense convection has shifted closer to the trough. The enhanced convective signal continues to move into the eastern Atlantic on day 1.5 (Fig. 3d) and the circulation shows a pronounced northeast-southwest tilt, indicative of stronger barotropic growth over the ocean compared to over land. The enhanced convection associated with the wave pattern has been displaced into the southerly flow behind the trough on day 1.5. A similar tilt and convective location with respect to the trough persists as the disturbance tracks northwestward on day 3 (Fig. 3e).

The troposphere-deep structure capture by the $T_{b}$ EOF can be seen in Fig. 4. The wind fields at $200 \mathrm{hPa}$ (Fig. 4) are approximately opposite to the circulations at midlevels (cf. Fig. 3). The peak convection corresponds to a mid- to lower-level cyclonic flow and an upper-level anticyclonic flow (Figs. 3c and 4). Consistent with the influence of convection, the upper-level circulation exhibits a marked divergent outflow that reaches as far south as $20^{\circ} \mathrm{S}$. This upper-level structure of $T_{b}$ EOF will be contrasted against the v700 EOF in section $5 \mathrm{~b}$. 

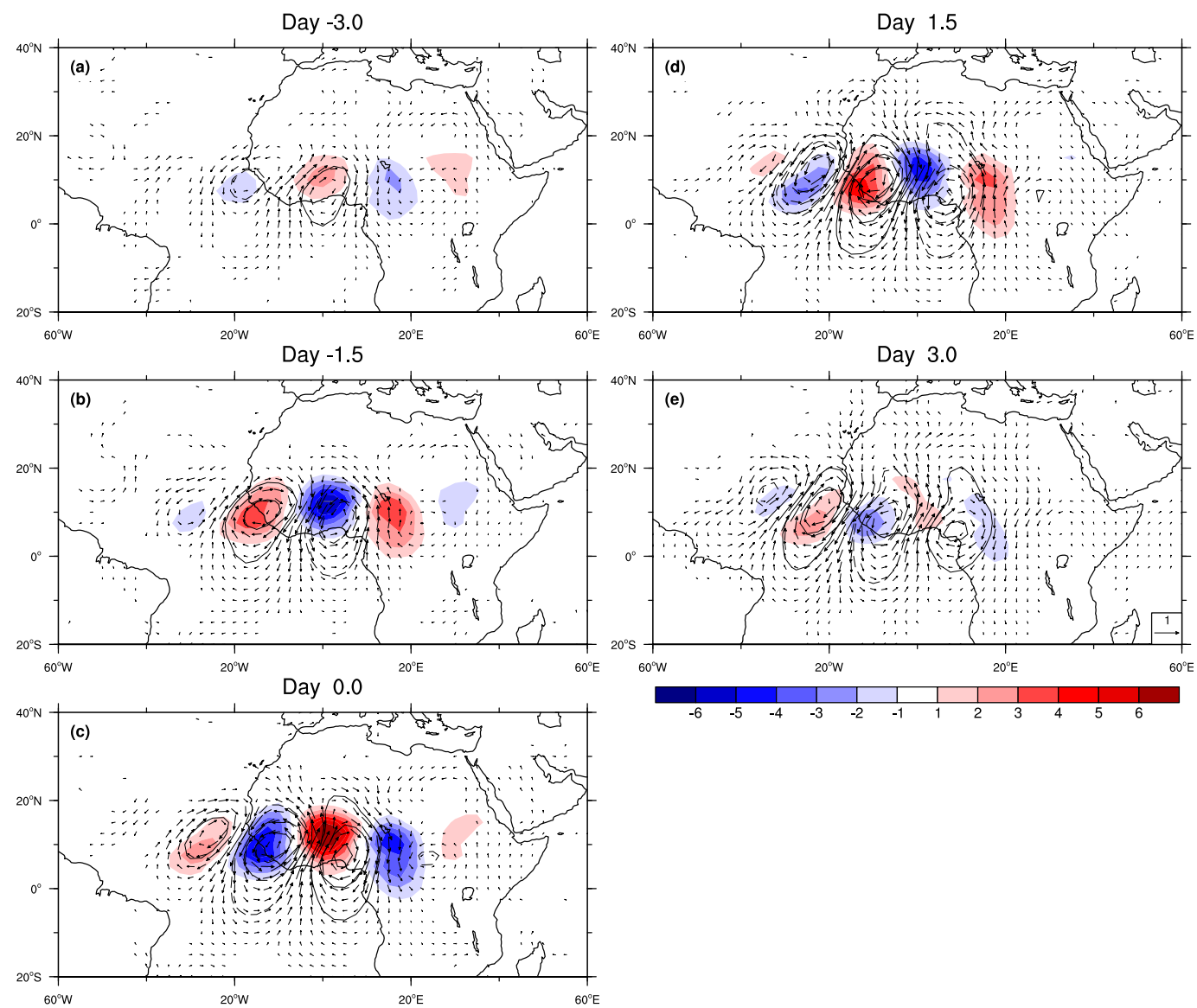

FIG. 3. Anomalous $T_{b}, 700$-hPa streamfunction, and wind regressed onto PC1 of $T_{b}$ EOF on (a) day -3 , (b) day -1.5 , (c) day $0,(\mathrm{~d})$ day 1.5 , and (e) day 3 . The amplitude was scaled to one standard deviation of PC1. The blue (red) shading denotes a negative (positive) $T_{b}$ perturbation. The contour interval of the streamfunction is $2 \times 10^{5} \mathrm{~m}^{2} \mathrm{~s}^{-1}$ with zero contours omitted. The anomalous wind vectors at $700 \mathrm{hPa}$ are also overlaid with a reference wind vector of $1 \mathrm{~m} \mathrm{~s}^{-1}$ at the bottom-right corner of (e). Only locally statistically significant $T_{b}$ at the $95 \%$ level is shown. Wind vectors are plotted if either the zonal or the meridional component is significant at the $95 \%$ level.

Figure 5 shows the zonal-height cross sections of meridional wind along $10^{\circ}$ and $15^{\circ} \mathrm{N}$. The vertical structures show a first baroclinic mode with minimal tilt. These vertically stacked structures are consistent with a convectively coupled wave where the mid- to lowerlevel flow is primarily opposite to that at the upper levels (Figs. 3 and 4). A close inspection reveals the $15^{\circ} \mathrm{N}$ cross section (Fig. 5b) has a weak eastward tilt below the AEJ level and westward above. This configuration is oriented against the vertical wind shear and can be related to the baroclinic zone along the Sahel. However, the weak tilt suggests a weak NV and strong contribution from convection (cf. cross sections of v700 EOF in section 5b).

\section{Horizontal structure of v700 EOF}

The v700 EOF is presented in Figs. 2c and 2d. The first two EOFs account for nearly $20 \%$ of the variance and are significantly separated from the rest using the rule from North et al. (1982). Although the same criterion also shows that EOF1 and EOF2 are separated from each other, the similarity of the two patterns and the

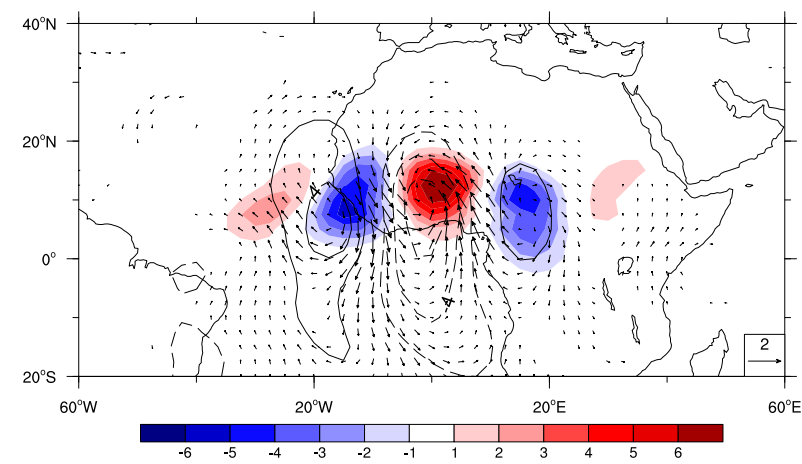

FIG. 4. As in Fig. 3, but for 200-hPa circulations on day 0. 

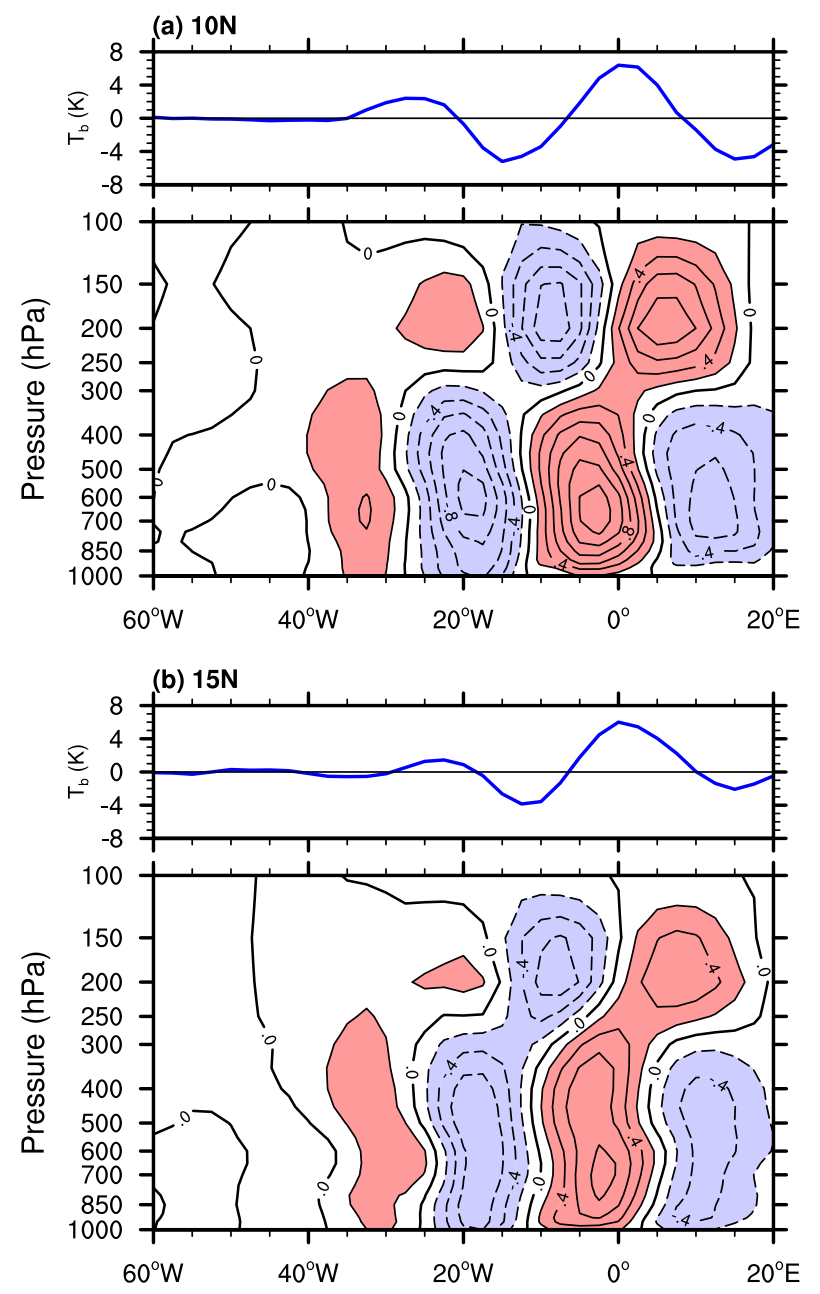

FIG. 5. Zonal-height cross sections of anomalous meridional wind of the $T_{b}$ EOF along (a) $10^{\circ}$ and (b) $15^{\circ} \mathrm{N}$. The contour interval is $0.2 \mathrm{~m} \mathrm{~s}^{-1}$. The $T_{b}(\mathrm{~K})$ along the same latitude is shown as a line plot above each panel.

maximum correlation coefficient of 0.92 at 1-day lag with PC2 leading indicate that the two form a quadrature pair, and depicts a westward-propagating wave with a period of about 4 days. The two EOFs highlight alternating patterns of opposite wind directions across northern Africa into the Atlantic as for the $T_{b}$ EOF. A northward shift of the peak wind perturbations is observed from West Africa to the Atlantic (Figs. 2c and $2 \mathrm{~d}$ ), consistent with a slight northwest tilt of the axis of peak 2-6-day activity across the Africa-Atlantic sector (Fig. 1b).

\section{a. Convective and mid-to lower-level structure}

The convection and circulation from the v700 EOF are shown in Fig. 6. At the simultaneous lag (Fig. 6c), the cyclonic circulation is collocated with the most intense convection around the West African coast. Pronounced horizontal tilts are seen on the flanks of the AEJ, suggestive of barotropic growth. The wave has a zonal wavelength of $3500 \mathrm{~km}$ at $10^{\circ} \mathrm{N}$. As for the $T_{b} \mathrm{EOF}$, a faster phase speed over the ocean compared to land is evident. The convective signal moves westward at an average of $11.8 \mathrm{~m} \mathrm{~s}^{-1}$ over Africa and $7.0 \mathrm{~m} \mathrm{~s}^{-1}$ over the Atlantic. Using meridional wind to track the wave, phase speeds of 13.5 and $7.9 \mathrm{~m} \mathrm{~s}^{-1}$ are obtained for the respective regions. These wave structure and characteristics are consistent with the AEW literature. Therefore the v700 EOF captures the main features of AEWs.

A closer look at the convective signals in the v700 EOF reveals a complicated "checkerboard" structure of convection between the Greenwich meridian and the West African coast (Fig. 6c). Convection is enhanced within the southern portion ahead of the coastal trough and suppressed in the northern part while the opposite is seen ahead of the following ridge. Although the convective anomalies along the Sahel are weaker than farther south, the elevated cloud tops correspond to increased precipitation in the TRMM dataset (not shown) and the signal becomes increasingly strong farther west (Fig. 6d). Using a slightly different technique of extended EOFs and compositing, Lau and Lau (1990) showed a structure resembling the dipole pattern seen here (cf. their Fig. 22b), but it was not emphasized in their work and other previous studies. The increased cloudiness is collocated with the moistening in the low levels associated with the southerlies of NV in the Sahel [see section 5a(2) and Fig. 10]. This favorable environment ahead of the ridge also appears consistent with the observed secondary peak of squall-line generation in the low-level southerlies in the Sahel (Duvel 1990; Fink and Reiner 2003).

Figure 6 also shows the evolution of the wave structure of the v700 EOF. The wind perturbation associated with the coastal trough on day 0 was first detected about 5 days before in East Africa around $0^{\circ}-10^{\circ} \mathrm{N}, 30^{\circ}-40^{\circ} \mathrm{E}$ with convection developing shortly after (not shown). By day -3 , the circulation is well defined and coupled to convection. As the wave moves across the Greenwich meridian, the enhanced convection extends eastward into the southerly flow in the Sahel region (Fig. 6b). By day 0, the checkerboard pattern of convection is established. As the system tracks over the Atlantic, the meridional convective dipoles weaken but persist (Figs. 6d and 6e), and the deepest convection moves into the southerly flow of the trough. The strongly tilted circulation associated with the AEW, though centered around $20^{\circ} \mathrm{N}$, reaches as far as northeastern South America where it can potentially influence the weather there (Figs. 6d,e). 

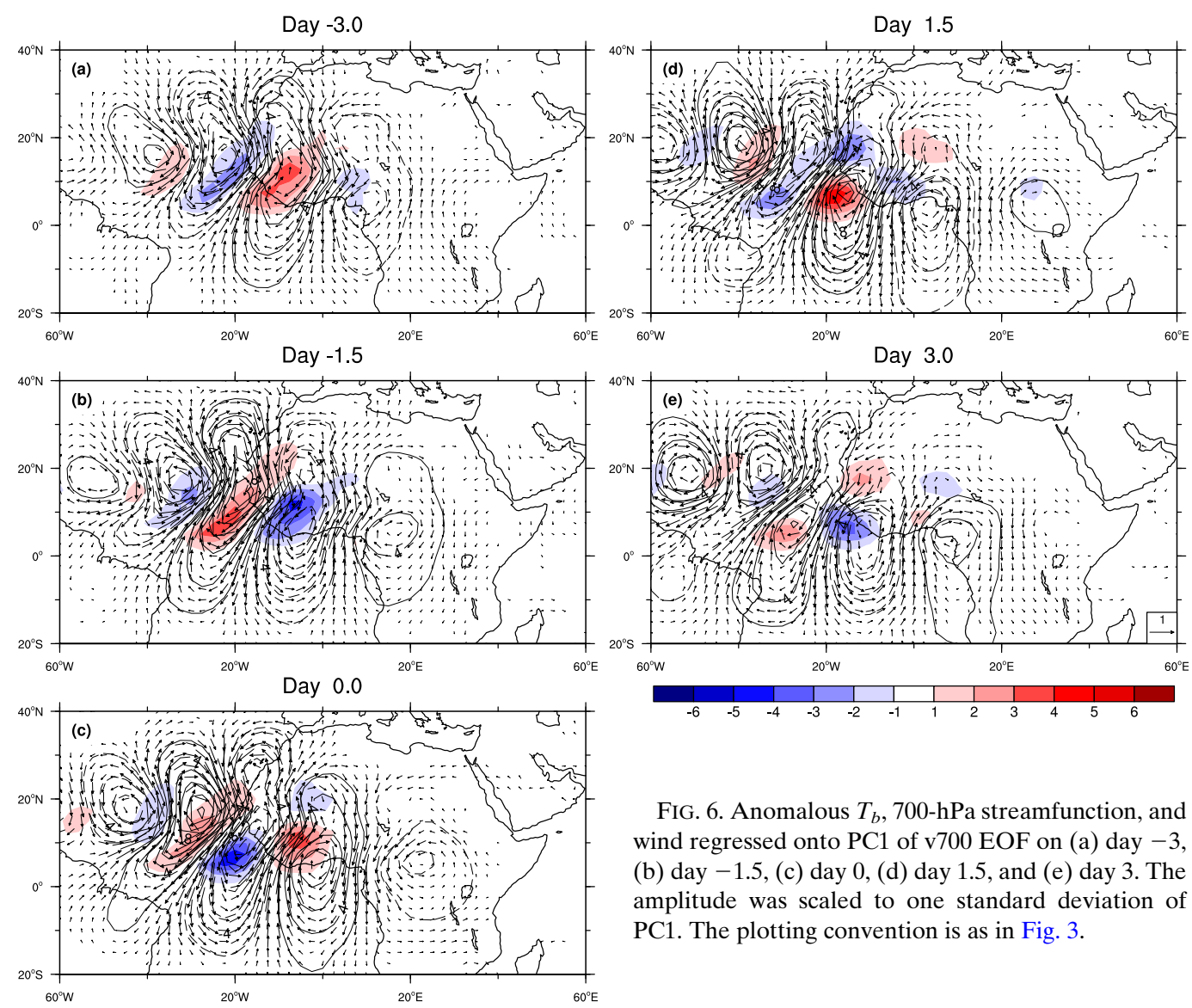

FIG. 6. Anomalous $T_{b}, 700$-hPa streamfunction, and wind regressed onto PC1 of v700 EOF on (a) day -3, (b) day -1.5 , (c) day 0 , (d) day 1.5 , and (e) day 3 . The amplitude was scaled to one standard deviation of PC1. The plotting convention is as in Fig. 3.

A striking feature of the v700 EOF compared to the $T_{b}$ EOF is its broad meridional extent (Fig. 6). The statistically significant wind perturbation associated with the v700 EOF extends from $20^{\circ} \mathrm{S}$ to $40^{\circ} \mathrm{N}$, substantially larger than the $T_{b}$ EOF. This meridionally broad extent is consistent with the meridionally broad synoptic activity in 700-hPa meridional wind (Fig. 1b). A physical argument for such broad meridional scale is the large Rossby radius of deformation in the tropics due to the small Coriolis parameter. This would account for the far-field impact of AEW-related vorticity anomalies on winds in the subtropical and equatorial regions. Although the meridional scale of AEWs and their potential interactions with those regions have been mentioned in the literature (e.g., KTH2006; Berry et al. 2007; Zawislak and Zipser 2010; Yang et al. 2018), the consequences of this have not been fully explored and explained. The following discussions will be devoted to understanding the meridional wave structure of the v700 EOF by emphasizing the AEW interactions with the equatorial and subtropical regions.

\section{1) AEW-EQuatorial interactions}

The v700 EOF circulation in the equatorial region is characterized by marked cross-equatorial flow. The associated geopotential height anomalies have an antisymmetric signature across the equator: strong and tilted toward the northeast in the north while weak and much less tilted in the south (Fig. 7). This geopotential height pattern spanning from central East Africa across the Atlantic to South America persists from day -6 to day 6 in our lag regression. In addition, these geopotential height patterns in the Southern Hemisphere remain equatorially trapped (centered about $10^{\circ} \mathrm{S}$ ) when the AEWs track northeastward over the ocean. For the divergence field, there is a maximum in divergence ahead of the 700-hPa trough and convergence behind between $5^{\circ}$ and $20^{\circ} \mathrm{N}$ (Fig. 7). This signature is mostly due to a combination of convective heating and dry dynamics associated with the AEWs (Fig. 6c). A structure of opposite sign of convergence and divergence, though much weaker, is present in the corresponding southern parts of the wave. The anomalous cross-equatorial meridional wind, antisymmetric geopotential height as well as divergence structure of the v700 EOF appears to resemble a mixed Rossby-gravity wave (MRG) from the equatorial mode solution in Matsuno (1966). Although the geopotential height anomalies to the south are weak compared to the north, similar values have been reported 


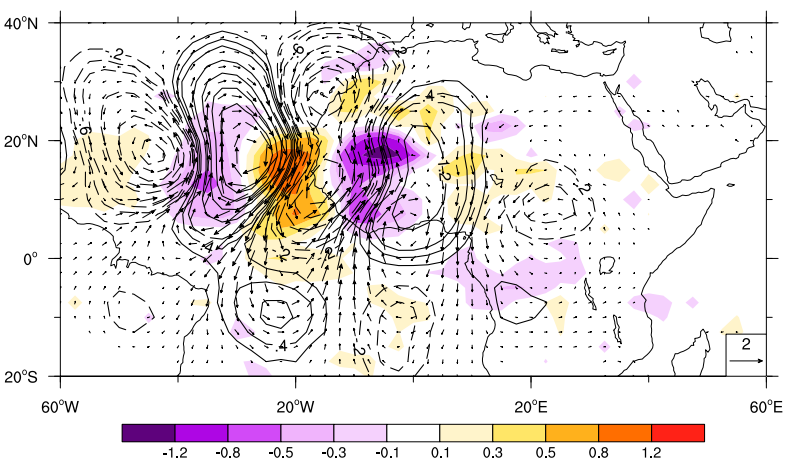

FIG. 7. As in Fig. 6, but for anomalous geopotential height (m; contours) and divergence $\left(10^{-6} \mathrm{~s}^{-1}\right.$; shading). The geopotential height is contoured every $0.2 \mathrm{~m}$ when the value is below $0.6 \mathrm{~m}$ and every $0.6 \mathrm{~m}$ when the value is above $0.6 \mathrm{~m}$. The zero line is omitted.

for MRGs in the central Pacific, where MRGs in the troposphere typical have their strongest signals (Liebmann and Hendon 1990). The maximum closed contour south of the equator is $0.6 \mathrm{~m}$ at $700 \mathrm{hPa}$ in Fig. 7, comparable to Fig. 12 of Liebmann and Hendon (1990) with a closed contour of $0.6 \mathrm{~m}$ at $850 \mathrm{hPa}$. The wave structure of the v700 EOF has the features of an AEW such as the horizontal tilts and convection location that are typical of AEWs, but it also possesses characteristics such as cross-equatorial flow and antisymmetric geopotential height in the equatorial region that resemble an MRG.

Motivated by this "hybrid" AEW-MRG structure found in the v700 EOF, we calculated a series of EOFs designed to isolate a "pure" MRG signal by placing the domains around the equator where the MRG signal of meridional wind maximizes. The zonal borders were fixed to be $40^{\circ} \mathrm{W}-40^{\circ} \mathrm{E}$, and the meridional boundaries varied from just at the equator to $20^{\circ} \mathrm{N}$ and $20^{\circ} \mathrm{S}$ with a $2.5^{\circ}$ interval. The tests were carried out on 2-6-dayfiltered meridional wind at 700 and $850 \mathrm{hPa}$. As an example, Fig. 8 shows the $700-\mathrm{hPa}$ circulation and convection associated with the EOF of $850-\mathrm{hPa}$ meridional wind with meridional boundaries of $15^{\circ} \mathrm{S}-15^{\circ} \mathrm{N}$. The main features, including the convective patterns, horizontal tilts, and cross-equatorial meridional winds, are almost identical to Fig. $6 c$ from the v700 EOF. The results from this MRG-targeted EOF test, regardless of the domain sizes, all show a close resemblance to the kinematic and convective fields obtained from the v700 EOF, suggesting a similar hybrid structure. The fact that both AEW and MRG-targeted EOFs show similar hybrid circulation highlights the coexistence and prevalence of $\mathrm{AEW}$ and MRG structure over the region.

While the Africa-Atlantic sector has been largely neglected in previous MRG studies, Liebmann and Hendon (1990) have attempted to investigate equatorially

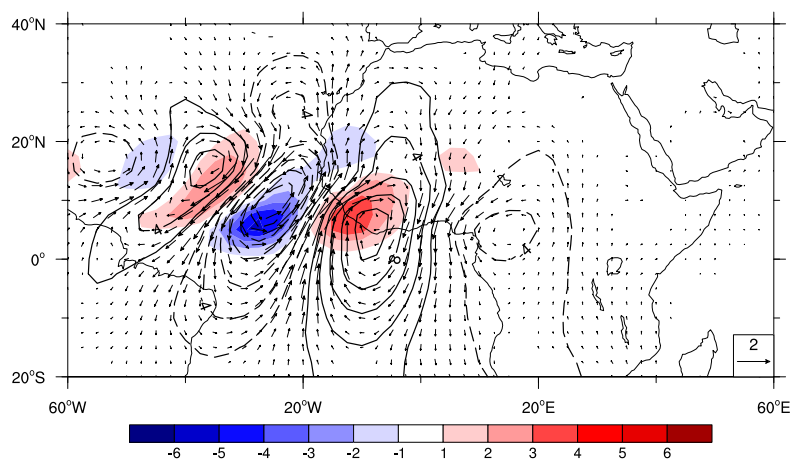

FIG. 8. Anomalous $T_{b}, 700-\mathrm{hPa}$ streamfunction, and wind regressed onto PC1 associated with the EOFs of 2-6-day-filtered meridional wind at $850 \mathrm{hPa}$ on day 0 . The EOF domain is $15^{\circ} \mathrm{S}-15^{\circ} \mathrm{N}, 40^{\circ} \mathrm{W}-40^{\circ} \mathrm{E}$ to target the peak meridional signals associated with MRGs. The plotting convention is as in Fig. 3.

trapped synoptic waves in the transition season (SeptemberDecember). They found that over the Atlantic, the odd number mode (i.e., MRG), despite the weak amplitude, has both wind and convective structure in agreement with the linear theory (their Fig. 13). In a regional climate simulation study, Hsieh and Cook (2007) speculated on the possibility that some disturbances over Africa and the tropical Atlantic may possess hybrid TDtype and MRG structures by the proximity of the TD power to the MRG in the wavenumber-frequency spectrum. More recently, Yang et al. (2018) proposed that AEWs could be amplified in the presence of upper-level MRGs and this amplification mechanism was thought to explain years of strong AEW activity. These studies provide evidence of the MRG activity over Africa and lend confidence to our result of MRG characteristics in the v700 EOF.

\section{2) AEW-SUBTROPICAL CIRCULATIONS}

Another unique feature captured by the v700 EOF is a vortex that forms off the coast of Morocco. This "Moroccan vortex" establishes as a cold-core circulation with a distinct PV perturbation as an AEW trough approaches the West African coast (Fig. 9). The feature can be traced back to day -1.5 when the anomalous northerlies associated with the AEW trough appear along the Moroccan coast and a weak PV perturbation start to develop (Fig. 9a). The southwestward-moving vortex rapidly intensifies as it traverses along the northwest African coast. By day 0, the intensity of the $\mathrm{PV}$ anomaly associated with the Moroccan vortex is just slightly weaker than that of the convectively coupled AEW to the south (Fig. 9b). Once the AEW trough moves over the Atlantic, the Moroccan vortex quickly weakens, and the two PV anomalies merge into one coherent structure (Fig. 9c). 

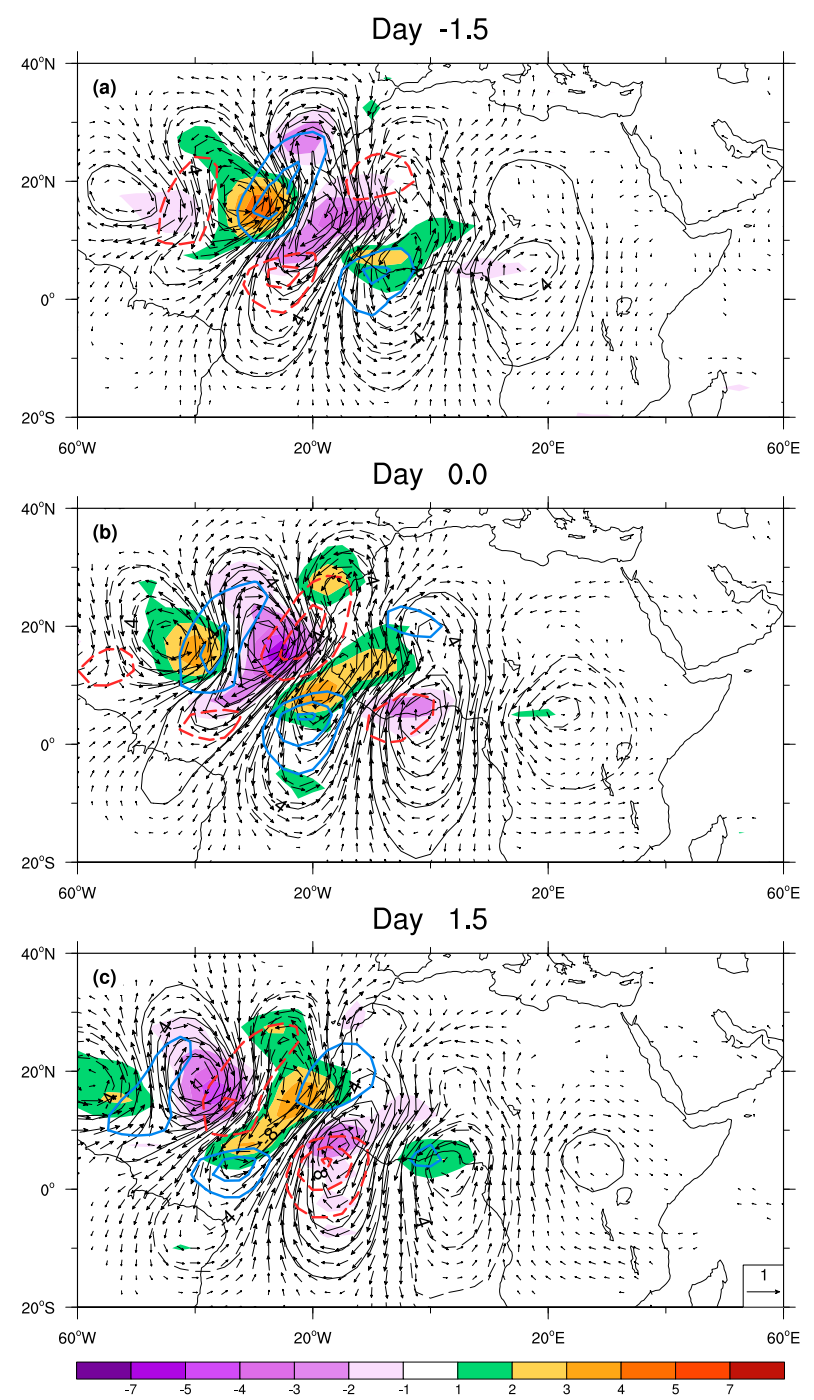

FIG. 9. Anomalous $700-\mathrm{hPa} \mathrm{PV}\left(10^{-2} \mathrm{PVU}\right)$, streamfunction, and wind regressed onto PC1 of v700 EOF on (a) day -1.5, (b) day 0 , and (c) day 1.5. Positive (negative) anomalies of specific humidity $\left(\mathrm{g} \mathrm{kg}^{-1}\right)$ are also contoured in solid blue (red) lines with an interval of $0.2 \mathrm{~g} \mathrm{~kg}^{-1}$ and zero contours omitted. The rest of plotting convention is as in Fig. 3.

The formation of the Moroccan vortex is thought to originate from the interaction of AEW and the basic state. A sharp PV gradient is located off the coast of subtropical West Africa in association with the semipermanent trough (Fig. 1c). When the wind anomalies associated with an AEW reach the coastal region, the $\mathrm{PV}$ gradient is perturbed resulting in a positive PV anomaly of the Moroccan vortex. In addition, from an instability perspective, as discussed in Thorncroft and Hoskins (1994), the negative meridional PV gradient of the jet presents three possible interactions satisfying the Charney-Stern instability criterion, with the equatorial and poleward flanks of the AEJ as well as the surface baroclinicity along the Sahel. Based on a longitudinal average of PV cross section (Fig. 2b of Reed et al. 1977), it was expected by the authors that the stronger PV gradient on the equatorward flank of the jet favors stronger wave growth south of the jet. However, as shown in Fig. 1c, the PV gradient poleward of the jet is present near the coast and over the Atlantic associated with the coastal trough. Although the geometry is more complex than that of a zonally symmetric basic state (e.g., Thorncroft and Hoskins 1994), it can be hypothesized that the opposite sign of meridional PV gradient of the AEJ and subtropical trough can enhance barotropic growth along the coast and contribute to the growth of the Moroccan vortex. Consistent with such a growth mechanism, we would also expect contemporaneous amplification of the wave equatorward of this.

It should be highlighted that the Moroccan vortex is different from the commonly recognized NV propagating along the Sahel. The NV forms in conjunction with the baroclinicity on the southern fringe of the desert and has peak intensity between 925 and $850 \mathrm{hPa}$. The NV circulations can be seen centered around $15^{\circ}-20^{\circ} \mathrm{N}$ and strengthen as they move westward toward the coast (Fig. 10). The warm-core structure is associated with a positive PV anomaly with circulation tilted eastward with height, suggestive of baroclinic growth (not shown). As the AEW trough approaches the coast, the PV associated with the NV and Moroccan vortex undergoes a complicated merging. On day -1.5 , the PV anomaly of the NV is located around $18^{\circ} \mathrm{N}, 10^{\circ} \mathrm{W}$ (Fig. 10a) while a separate weak $\mathrm{PV}$ perturbation associated with the Moroccan vortex starts to develop at approximately $30^{\circ} \mathrm{N}, 12^{\circ} \mathrm{W}$ at $700 \mathrm{hPa}$ (Fig. 9a) and $850 \mathrm{hPa}$ (Fig. 10a). By day 0, the PV of the NV first merges with the PV of the convectively active southern wave (Fig. 10c). The PV of the Moroccan vortex becomes a southwest-northeast-oriented strip (Figs. 9b and 10b) and quickly aggregates with the southern PV anomaly (Fig. 10c). This evolution demonstrates the different origins of the NV and Moroccan vortex and highlights the PV merging when an AEW trough moves into the Atlantic.

Both the NV and the Moroccan vortex appear to be important for the presence of lower- to midtroposphericlevel dry air ahead of an AEW trough around and north of the AEJ. In association with the two circulations, specific humidity (Figs. 9 and 10) reveals that dry air can originate from both the desert and subtropics. The northeasterly winds associated with the NV contribute to the moisture deficit ahead of the low-level trough while the northwesterly winds associated with the Moroccan vortex draws in midlevel dry air from farther north. Brammer and Thorncroft (2017) found that as an AEW leaves the coast, the northwesterly flow from the mid-lower levels becomes increasingly important. This 
Day -1.5
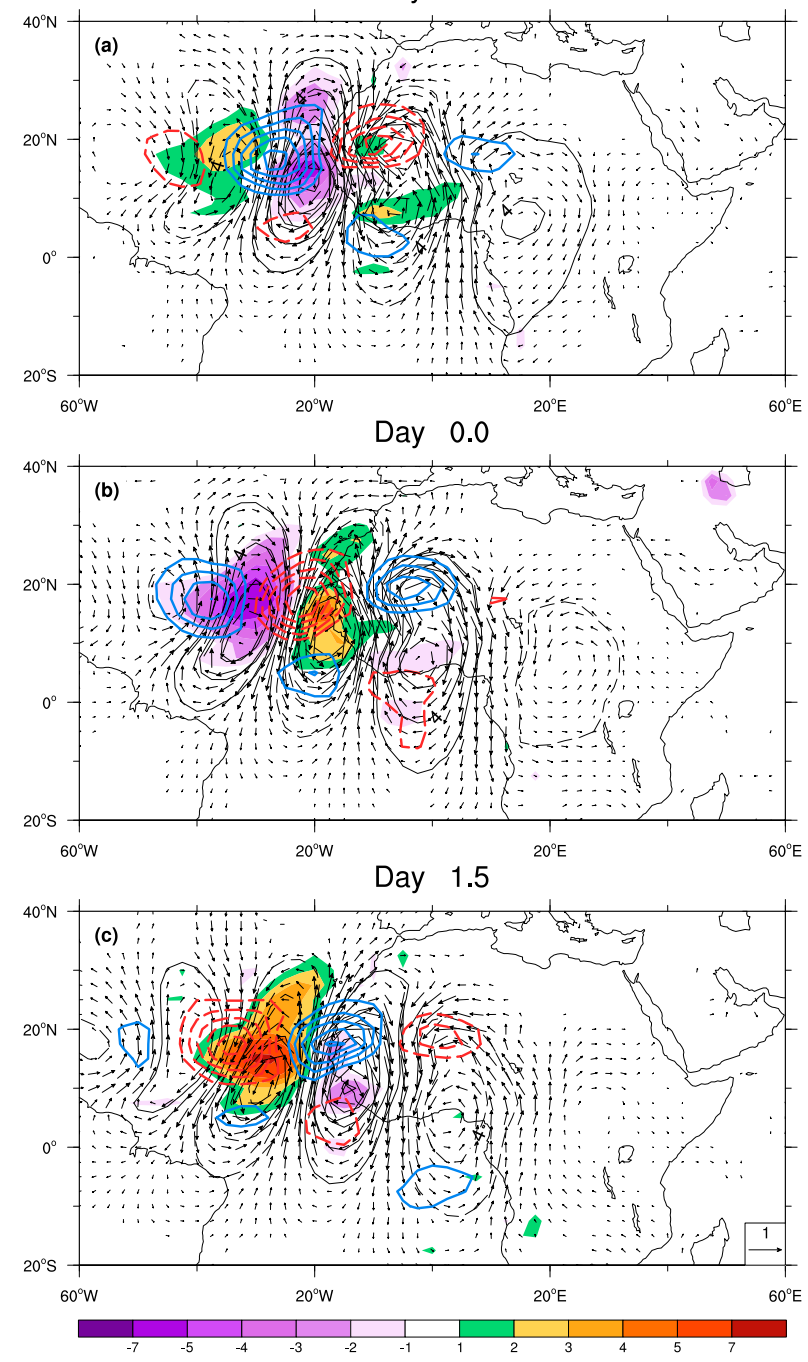

FIG. 10. As in Fig. 9, but at $850 \mathrm{hPa}$.

is consistent with the northwesterly flow contributed by the Moroccan vortex here and suggests that the Moroccan vortex can have an impact on the evolution and structure of the wave in some cases.

\section{b. Upper-level structure}

The structure of the v700 EOF at $200 \mathrm{hPa}$ encompasses both equatorial and subtropical regions. At simultaneous lags along $20^{\circ} \mathrm{W}$ (Fig. 9c), the upper-level flow is characterized by pairs of clockwise and counterclockwise circulations, centered along the equator and around $25^{\circ} \mathrm{N}$. Tracing the equatorial circulation back to day -3 , it can be seen straddling $0^{\circ}, 5^{\circ} \mathrm{E}$ (Fig. 11a), where it is associated with weakly enhanced convection in the westerlies. This circulation is first detected at day -5 around $40^{\circ} \mathrm{E}$ around the equator (not shown). As the disturbance moves westward
(Figs. 11b and 11c), the convection on the northern side strengthens and the equatorial wind perturbation rapidly intensifies and undergoes marked zonal-scale contraction. The half wavelength, as measured from peak southerly to northerly at the equator, shrinks from $2500 \mathrm{~km}$ on day -3 east of the Greenwich to $1700 \mathrm{~km}$ on day 0 near the West African coast. The equatorial disturbance seen at day -3 (Fig. 11a) and before (not shown) resembles an upper-level MRG circulation. This analysis suggests that MRGs may often be associated with AEWs, a result that is further studied in Y. M. Cheng et al. (2019, unpublished manuscript).

In the subtropics, the 200-hPa circulations are associated with convective anomalies in the Sahel and the subtropical PV gradient (not shown). The alternating northerlies and southerlies north of $20^{\circ} \mathrm{N}$ are associated with divergence and convergence (not shown) collocated with enhanced and suppressed convection in the Sahel (Fig. 11c). We hypothesize that the AEW-associated convection results in a sinusoidal outflow (southerly) and inflow (northerly) anomaly at $200 \mathrm{hPa}$ that perturbs the subtropical PV gradient resulting in a subtropical Rossby wave train over North Africa and the Mediterranean. Consistent with this finding, a similar subtropical wave structure at the upper levels is seen at the base point of $15^{\circ} \mathrm{N}, 17.5^{\circ} \mathrm{W}$ in $\mathrm{KTH} 2006$, where the convection also extends into the Sahel. The formation of this wave train is similar to that of the Moroccan vortex at the midlevels in that the wind anomalies from the wave perturb the background PV gradient. The fact that these interactions are captured by EOF analysis suggests their frequent appearance in tandem with AEWs and thus should be regarded as an integral part of an AEW.

The different vertical structures linked to the $T_{b}$ EOF and v700 EOF are highlighted by comparing Figs. 5 and 12. The $T_{b}$ EOF shows a first baroclinic mode and a strong upper-level anticyclone at the peak convective signal (Fig. 5). In contrast, the v700 EOF shows a weak upper-level anticyclone associated with the convection (Fig. 12a) and a clear vertical tilt, particularly along $15^{\circ} \mathrm{N}$ (Fig. 12b). The wave tilts eastward with height below the jet and westward above. This vertical structure suggests a relatively greater contribution from baroclinic energy conversions to the growth of the v700 EOF AEW compared to diabatic energy contributions, which are likely more important for the $T_{b} \mathrm{EOF} \mathrm{AEW}$. The different vertical cross sections further demonstrate the structural differences captured by the $T_{b}$ and v700 EOFs.

\section{Summary and discussion}

In this study, the variability of AEWs is investigated using an EOF method to isolate the dominant wave 
Day -3.0
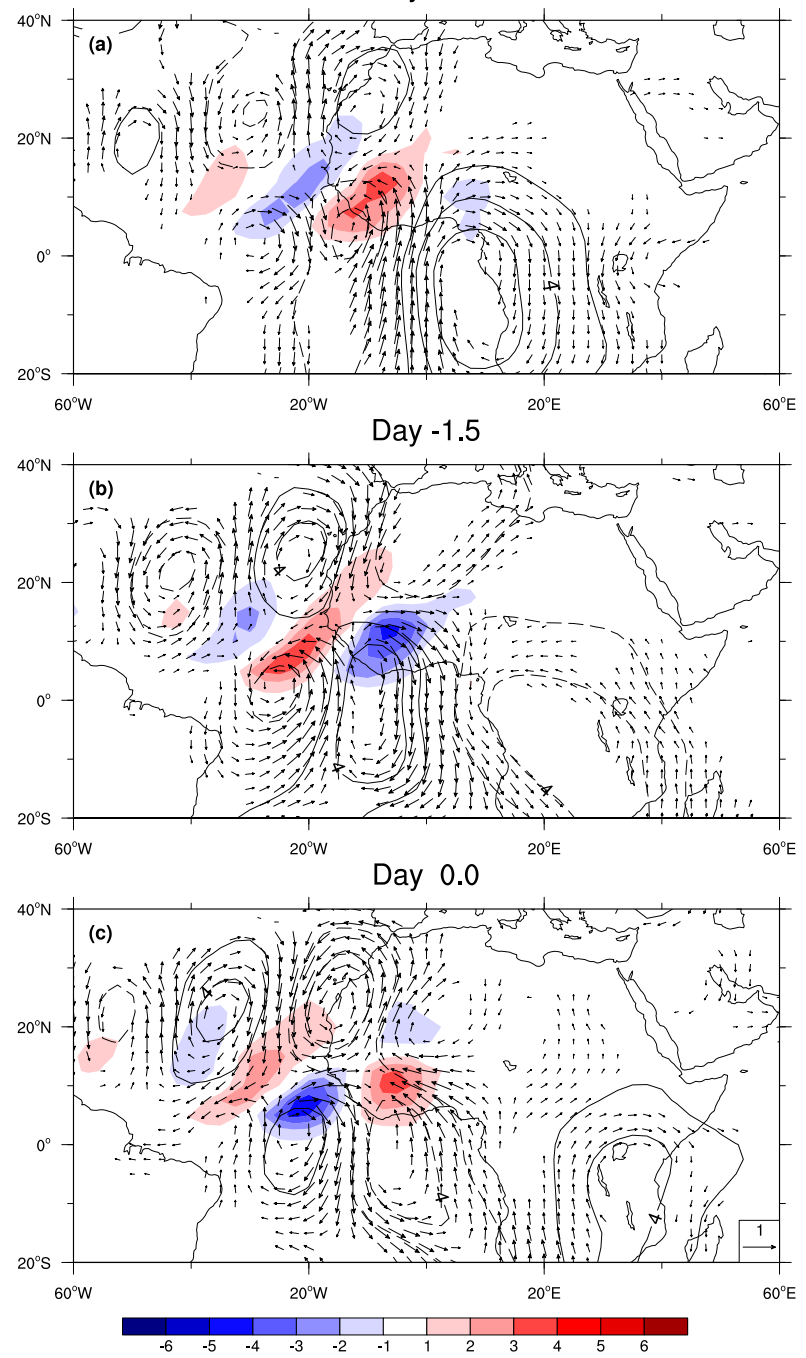

FIG. 11. Anomalous $T_{b}, 200-\mathrm{hPa}$ streamfunction, and wind regressed onto PC1 of v700 EOF on day 0 . The plotting convention is as in Fig. 6.

structures. The calculation was carried out with two commonly used variables to analyze AEWs: $T_{b}\left(T_{b}\right.$ $\mathrm{EOF})$ and meridional wind at $700 \mathrm{hPa}(\mathrm{v} 700 \mathrm{EOF})$. The structure and evolution associated with the two kinds of EOFs were studied through lag regression.

The regression map of the $T_{b}$ EOF depicts a structure and evolution similar to those documented in the previous AEW studies. The $T_{b}$ EOF shows circulations that have a strong association with convective anomalies. Except for a marked cross-equatorial flow at upper levels linked to the convective outflow and a weak northern vortex, the AEW circulations are primarily confined to convectively active areas south of the AEJ. The wave evolution denotes a typical shift of the deepest convection from northerlies to the trough and toward
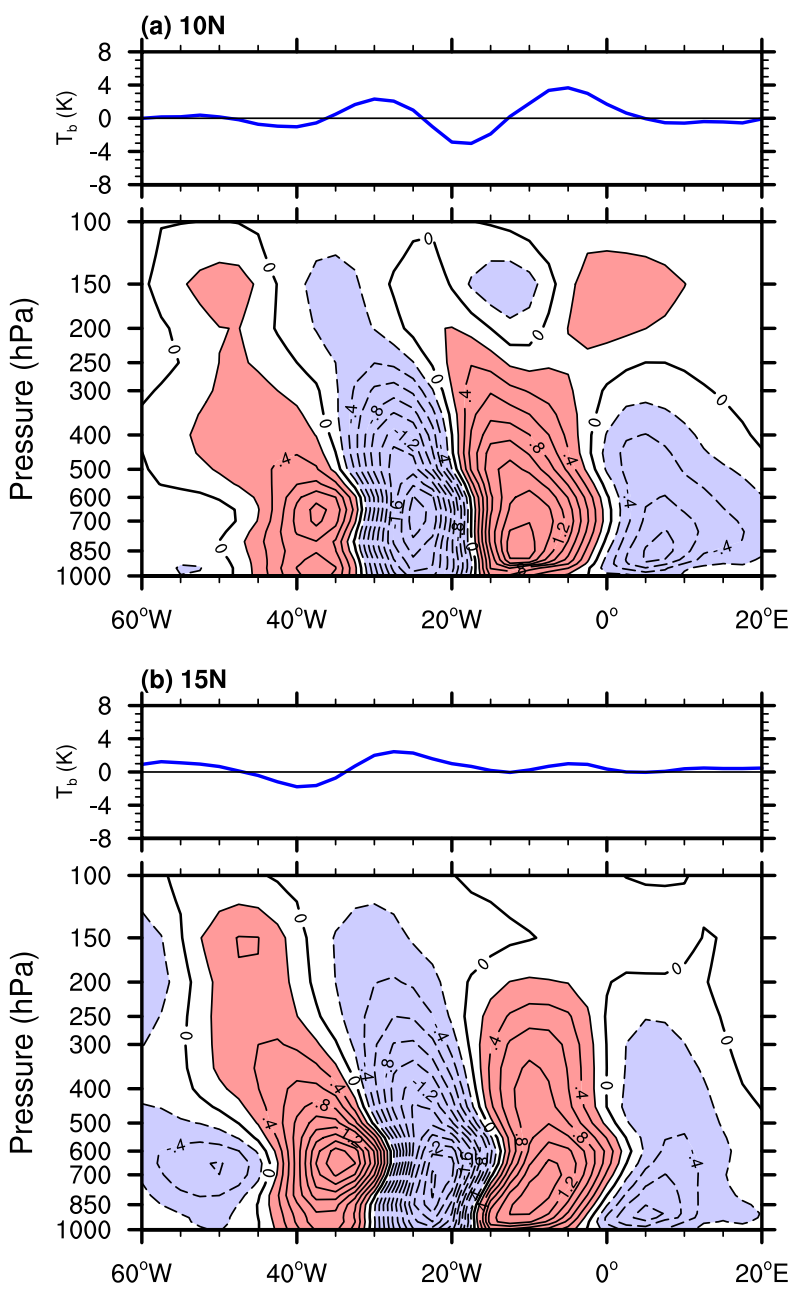

FIG. 12. Zonal-height cross section of anomalous meridional wind from v700 EOF along (a) $10^{\circ}$ and (b) $15^{\circ} \mathrm{N}$. The $T_{b}(\mathrm{~K})$ along the same latitude is shown as a line plot above each panel. The plotting convention is as in Fig. 5.

southerlies as the wave propagates from East Africa into the Atlantic. This structure is consistent with previous studies (e.g., Duvel 1990; KTH2006).

In contrast, the AEWs from the v700 EOF are highlighted by a complicated horizontal structure that has not been emphasized previously. First, the coupled convective signals are characterized by a horizontal checkerboard pattern. A meridional dipole of enhanced and suppressed convection around $10^{\circ}$ and $20^{\circ} \mathrm{N}$ is seen ahead of a trough and with the opposite dipole behind it. The southern convective signals are similar to those seen with the $T_{b}$ EOF as well as in the literature (e.g., Duvel 1990; KTH2006) while the northern signals in the Sahel are less well documented. The southerlies of the northern vortex moisten the low levels ahead of the $700-\mathrm{hPa}$ ridge and favor enhanced convection in the Sahel. This result is consistent with the secondary peak of squall-line 
formation in the region reported in previous studies (e.g., Fink and Reiner 2003).

The most striking feature of the v700 EOF is the broad meridional circulation. The wind perturbation spans from $20^{\circ} \mathrm{S}$ to $40^{\circ} \mathrm{N}$, substantially larger than most reported in the literature but consistent with KTH2006. This broad flow pattern highlights the interactions with the equatorial as well as subtropical regions. While the wave structure in the tropics shows a typical AEW signature, the circulation in the equatorial region is characterized by MRG-like kinematics. The cross-equatorial flow, antisymmetric geopotential height, as well as the convergence patterns, resemble an MRG structure. This "hybrid" between AEWs and MRGs motivated pursuit of a "pure" MRG in the Atlantic-Africa region using EOF domains centered at the equator. The results consistently demonstrate the hybrid structure regardless of the domain chosen. This inseparable nature of the AEW-MRG hybrid further confirms the prevalence and their overlapping dynamics in the region.

For the AEW-extratropical interactions in the v700 EOF, the principal mechanism appears similar throughout the troposphere: the wind anomalies associated with AEWs intermittently perturb the background PV gradient around $25^{\circ} \mathrm{N}$ resulting in a subtropical Rossby wave train. At upper levels, the wind anomalies are associated with the divergent outflow and inflow of the checkerboard-patterned convection. At mid- to lower levels, the wind anomalies associated with the AEW trough lead to an anomalous midlevel vortex off the Moroccan coast. The northerlies associated with this "Moroccan vortex" appear to draw midlevel dry air from subtropical regions into the AEW trough in the tropics. In addition, a complex merging of PV anomalies associated with the Moroccan vortex, northern vortex, and the convective southern wave takes place in the mid- to lower levels as an AEW trough approaching the West African coast. The aggregation of PV anomalies, as well as mixing of dry air and dust (Grogan and Thorncroft 2019) associated with those embedded circulations, can be important processes in determining the structure and evolution of each individual AEW over the Atlantic.

The presence of the AEW-MRG hybrid structure associated with the v700 EOF is one of the major findings in our study. As highlighted in KTH2006, the fact that AEWs are able to influence winds in the equatorial region is consistent with the possibility of interactions with other equatorially trapped modes, as was also recently investigated by Yang et al. (2018). The analysis presented here showed that the broad meridional wave structure associated with the v700 EOF appears to project onto the MRG. While this suggests that AEWs might be able to force MRG structures to the south, the opposite might also be possible. Convection and wind perturbations associated with preexisting MRGs may be able to influence AEWs. This could occur in association with convective triggering (cf. Berry et al. 2007; Thorncroft et al. 2008) or in association with wind perturbations in the vicinity of the AEJ (cf. Leroux et al. 2011). The direction of causality cannot be deduced using the EOF approach we have used here. We can conclude, however, that the AEW and MRG structures often overlap in the West African region. The direction of causality may vary from case to case and needs to be assessed with further analysis.

Consistent with our finding of the AEW-MRG hybrid, Yang et al. (2018) also found MRG activity over Africa but their work had a different emphasis. They focused on the influence of MRGs on the AEW intensity and sought to explain the triggering of MRGs by the subtropical Rossby waves in the Southern Hemisphere. It was argued that the enhanced upper-level divergence and southerly flow associated with the MRG could amplify the AEW through vorticity stretching. In contrast, in the current study, we emphasize the structure of the AEWMRG hybrid due to the interaction between AEWs and MRGs. Both studies provide complementary evidence of MRGs in Africa and their interaction with AEWs.

The present work provides new insights into two types of AEW behavior. One has limited spatial extent and a first baroclinic mode in the vertical while the other has a broad meridional extent interacting with equatorial and subtropical waves and an eastward-tilting structure in the vertical below the AEJ. Because of the use of two different variables (i.e., $T_{b}$ and $700-\mathrm{hPa}$ meridional wind), the obtained waves are inevitably biased toward structures better correlated with the index itself. Nonetheless, these contrasting meridional scales appear consistent with the results of Berry et al. (2007). The limited meridional extent of $T_{b}$ EOF (Fig. 3) aligns with the subset of waves that lack a coherent northern vortex structure in their study. These waves were thought to grow predominantly through diabatic processes and weakly coupled to the basic state. In contrast, the broad cross-jet structures associated with the v700 EOF show prominent horizontal and vertical tilts. These structures were hypothesized to primarily develop through barotropic and baroclinic energy conversions through Rossby wave dynamics. The differences in wave growth mechanism are consistent with the vertical structure derived from the two EOFs in this study.

To explore the impact of different AEW behaviors depicted by the different EOFs, composites of TRMM precipitation based on each pair of PCs are shown in Fig. 13. The full-resolution TRMM data were used to 

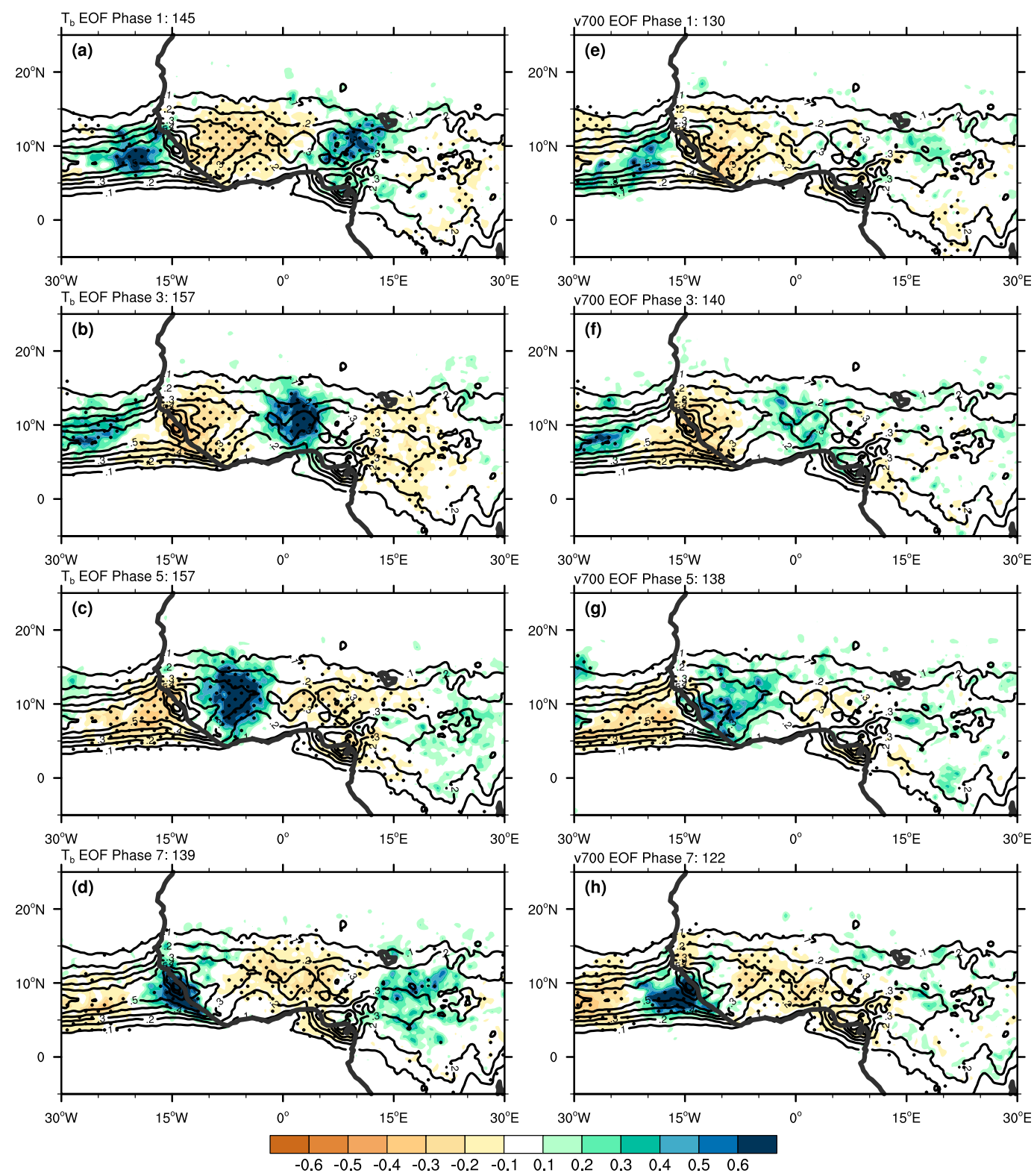

FIG. 13. Composite of anomalous TRMM rainfall ( $\mathrm{mm} \mathrm{h}^{-1}$; colors) for (a)-(d) the $T_{b}$ EOF and (e)-(h) the v700 EOF. The anomalies are based on the climatology of July-September 1998-2013 (contours; plotted every $0.1 \mathrm{~mm} \mathrm{~h}^{-1}$ ). The stippling denotes where the rainfall is significantly different from the climatology at the $95 \%$ confidence interval using two-tailed $t$ test. The AEW phases are arbitrarily chosen to geographically align the precipitating areas (approximate trough position) in the two EOFs. The number of time steps used to composite each phase is noted above each panel.

reveal finescaled structure in rainfall patterns. With the passage of waves identified by the $T_{b}$ EOF and v700 EOF, there are alternating enhanced and suppressed precipitation areas. As in convective signals for the two kinds of EOFs (Figs. 3 and 6), the $T_{b}$ EOF captures AEWs with higher rainfall rate and more compact pattern while the v700 EOF depicts AEWs with lower precipitation rate but impacting a more widespread area. From an operational forecasting point of view, one could gain insight in the expected rainfall rates and patterns when different modes are active. For example, during a v700 EOF active period, the rainfall pattern is typically scattered and relatively weak east of the prime meridian. However, there are scattered rainfall peaks north of $15^{\circ} \mathrm{N}$ throughout each phase, consistent with the secondary peak of convection in the checkerboard 
pattern of the v700 EOF. These northern rainfall anomalies are particularly notable along the west coast, where they tend to have opposite signs from the ones farther south (e.g., Figs. 13e,f,h). Although those precipitation anomalies are relatively weak and not statistically significant in the composites, they may be important for the semiarid Sahel region for particular high-amplitude cases. Indeed, the 20-day wet period in the Sahel regions identified by Lafore et al. (2017) was associated with strong projection onto the v700 EOF over 5 days. The MRGs were shown to reinforce the low-level monsoon flow and help amplify the AEWs. During the period, heavy precipitation events caused flooding and damage over a wide region of the Sahel. These results highlight the contribution of equatorial waves to rainfall in the West African monsoon (Schlueter et al. 2019a,b).

The results from the current study highlight two contrasting types of AEW behavior: one has compact convection with a confined circulation while the other has a meridionally broad circulation coupled with complicated convective signals and shows strong interactions with the subtropical and equatorial waves. These two types of AEWs provide a framework to objectively classify AEW structure based on their dynamic and thermodynamics characteristics. The fundamental question yet to be answered is, What is the cause for such structural variability? Studies have shown that the structure and intensity of AEWs are sensitive to the basic state such as the AEJ (e.g., Leroux and Hall 2009). Other idealized studies have shown that inclusion of the latent heat release in the ascending part of the AEWs lead to more intense waves with smaller zonal scales (e.g., Thorncroft and Hoskins 1994; Cornforth et al. 2009). Interactions with other synoptic waves, such as the upper-level MRG that will be discussed in Y. M. Cheng et al. (2019, unpublished manuscript), can also potentially modulate the structure of AEWs. Another important topic is how the wave structure variability is manifested in terms of intraseasonal and interannual AEW activity (e.g., Sultan et al. 2003; Grist et al. 2002). Such variability can be important for influencing the weather experienced in the tropical Africa as well as the nature and probability of tropical cyclone genesis in the Atlantic. It is also important to assess the extent to which such variability in AEW structures might be associated with long-term trends or decadal variations in the region (Ruti and Dell'Aquila 2010) and their impact on summer precipitation (Philippon et al. 2010).

Acknowledgments. We thank three anonymous reviewers for their comments, which helped clarify and improve the manuscript. We are also grateful for
Dr. Paul E. Roundy's input on MRGs. This work was supported by NSF Grants 1321568 and 1747844.

\section{REFERENCES}

Bain, C. L., D. J. Parker, N. Dixon, A. H. Fink, C. M. Taylor, B. Brooks, and S. F. Milton, 2011: Anatomy of an observed African easterly wave in July 2006. Quart. J. Roy. Meteor. Soc., 137, 923-933, https://doi.org/10.1002/qj.812.

Berry, G. J., and C. Thorncroft, 2005: Case study of an intense African easterly wave. Mon. Wea. Rev., 133, 752-766, https:// doi.org/10.1175/MWR2884.1.

,-- , and T. Hewson, 2007: African easterly waves during 2004-Analysis using objective techniques. Mon. Wea. Rev., 135, 1251-1267, https://doi.org/10.1175/MWR3343.1.

Brammer, A., and C. D. Thorncroft, 2017: Spatial and temporal variability of the three-dimensional flow around African easterly waves. Mon. Wea. Rev., 145, 2879-2895, https:// doi.org/10.1175/MWR-D-16-0454.1.

Burpee, R. W., 1975: Some features of synoptic-scale waves based on a compositing analysis of GATE data. Mon. Wea. Rev., 103, 921-925, https://doi.org/10.1175/1520-0493(1975)103<0921: SFOSWB $>2.0 . \mathrm{CO} ; 2$.

Carlson, T. N., 1969: Some remarks on African disturbances and their progress over the tropical Atlantic. Mon. Wea. Rev., 97, 716-726, https://doi.org/10.1175/1520-0493(1969)097<0716: SROADA $>2.3 . \mathrm{CO} ; 2$.

Cornforth, R. J., B. J. Hoskins, and C. D. Thorncroft, 2009: The impact of moist processes on the African easterly jet-African easterly wave system. Quart. J. Roy. Meteor. Soc., 135, 894-913, https://doi.org/10.1002/qj.414.

Dee, D. P., and Coauthors, 2011: The ERA-Interim reanalysis: Configuration and performance of the data assimilation system. Quart. J. Roy. Meteor. Soc., 137, 553-597, https://doi.org/ 10.1002/qj.828.

Diaz, M., and A. Aiyyer, 2013a: The genesis of African easterly waves by upstream development. J. Atmos. Sci., 70, 3492-3512, https://doi.org/10.1175/JAS-D-12-0342.1.

—, and 2013b: Energy dispersion in African easterly waves. J. Atmos. Sci., 70, 130-145, https://doi.org/10.1175/JAS-D-12-019.1.

Diedhiou, A., S. Janicot, A. Viltard, P. de Felice, and H. Laurent, 1999: Easterly wave regimes and associated convection over West Africa and tropical Atlantic: Results from the NCEP/NCAR and ECMWF reanalyses. Climate Dyn., 15, 795-822, https:// doi.org/10.1007/s003820050316.

Duchon, C. E., 1979: Lanczos filtering in one and two dimensions. J. Appl. Meteor., 18, 1016-1022, https://doi.org/10.1175/15200450(1979)018<1016:LFIOAT>2.0.CO;2.

Duvel, J. P., 1990: Convection over tropical Africa and the Atlantic Ocean during northern summer. Part II: Modulation by easterly waves. Mon. Wea. Rev., 118, 1855-1868, https://doi.org/ 10.1175/1520-0493(1990)118<1855:COTAAT $>2.0$. CO;2.

Fink, A. H., and A. Reiner, 2003: Spatiotemporal variability of the relation between African easterly waves and West African squall lines in 1998 and 1999. J. Geophys. Res., 108, 4332, https://doi.org/10.1029/2002JD002816.

Grist, J. P., S. E. Nicholson, and A. I. Barcilon, 2002: Easterly waves over Africa. Part II: Observed and modeled contrasts between wet and dry years. Mon. Wea. Rev., 130, 212-225, https://doi.org/ 10.1175/1520-0493(2002)130<0212:EWOAPI > 2.0.CO;2.

Grogan, D. F., and C. D. Thorncroft, 2019: The characteristics of African easterly waves coupled to Saharan mineral dust 
aerosols. Quart. J. Roy. Meteor. Soc., 145, 1130-1146, https:// doi.org/10.1002/qj.3483.

Hodges, K. I., D. W. Chappell, G. J. Robinson, and G. Yang, 2000: An improved algorithm for generating global window brightness temperatures from multiple satellite infrared imagery. J. Atmos. Oceanic Technol., 17, 1296-1312, https://doi.org/10.1175/ 1520-0426(2000)017<1296:AIAFGG > 2.0.CO;2.

Hsieh, J.-S., and K. H. Cook, 2007: A study of the energetics of African easterly waves using a regional climate model. J. Atmos. Sci., 64, 421-440, https://doi.org/10.1175/JAS3851.1.

Huffman, G. J., and Coauthors, 2007: The TRMM Multisatellite Precipitation Analysis (TMPA): Quasi-global, multiyear, combinedsensor precipitation estimates at fine scales. J. Hydrometeor., $\mathbf{8}$, 38-55, https://doi.org/10.1175/JHM560.1.

Janiga, M. A., and C. D. Thorncroft, 2013: Regional differences in the kinematic and thermodynamic structure of African easterly waves. Quart. J. Roy. Meteor. Soc., 139, 1598-1614, https:// doi.org/10.1002/qj.2047.

Kiladis, G. N., C. D. Thorncroft, and N. M. J. Hall, 2006: Threedimensional structure and dynamics of African easterly waves. Part I: Observations. J. Atmos. Sci., 63, 2212-2230, https:// doi.org/10.1175/JAS3741.1.

— J. Jias, and M. Gehne, 2016: The relationship between equatorial mixed Rossby-gravity and eastward inertio-gravity waves. Part I. J. Atmos. Sci., 73, 2123-2145, https://doi.org/ 10.1175/JAS-D-15-0230.1.

Lafore, J.-P., and Coauthors, 2017: A multi-scale analysis of the extreme rain event of Ouagadougou in 2009. Quart. J. Roy. Meteor. Soc., 143, 3094-3109, https://doi.org/10.1002/qj.3165.

Lau, K.-H., and N.-C. Lau, 1990: Observed structure and propagation characteristics of tropical summertime synoptic scale disturbances. Mon. Wea. Rev., 118, 1888-1913, https://doi.org/ 10.1175/1520-0493(1990)118<1888:OSAPCO > 2.0.CO;2.

$\ldots$, and _ 1992: The energetics and propagation dynamics of tropical summertime synoptic-scale disturbances. Mon. Wea. Rev., 120, 2523-2539, https://doi.org/10.1175/1520-0493(1992) $120<2523$ :TEAPDO $>2.0$. CO;2.

Leroux, S., and N. M. J. Hall, 2009: On the relationship between African easterly waves and the African easterly jet. J. Atmos. Sci., 66, 2303-2316, https://doi.org/10.1175/2009JAS2988.1.

,-- , and G. N. Kiladis, 2010: A climatological study of transient-mean-flow interactions over West Africa. Quart. J. Roy. Meteor. Soc., 136, 397-410, https://doi.org/10.1002/qj.474.

,$- \ldots$, and -2011 : Intermittent African easterly wave activity in a dry atmospheric model: Influence of the extratropics. J. Climate, 24, 5378-5396, https://doi.org/10.1175/JCLI-D-11-00049.1.

Liebmann, B., and H. H. Hendon, 1990: Synoptic-scale disturbances near the equator. J. Atmos. Sci., 47, 1463-1479, https://doi.org/ 10.1175/1520-0469(1990)047<1463:SSDNTE > 2.0.CO;2.

Livezey, R. E., and W. Y. Chen, 1983: Statistical field significance and its determination by Monte Carlo techniques. Mon. Wea. Rev., 111, 46-59, https://doi.org/10.1175/1520-0493(1983)111<0046: SFSAID $>2.0 . \mathrm{CO} ; 2$.

Matsuno, T., 1966: Quasi-geostrophic motions in the equatorial area. J. Meteor. Soc. Japan, 44, 25-43, https://doi.org/10.2151/ jmsj1965.44.1_25.

Mekonnen, A., C. D. Thorncroft, and A. R. Aiyyer, 2006: Analysis of convection and its association with African easterly waves. J. Climate, 19, 5405-5421, https://doi.org/10.1175/JCLI3920.1.

Monahan, A. H., J. C. Fyfe, M. H. P. Ambaum, D. B. Stephenson, and G. R. North, 2009: Empirical orthogonal functions: The medium is the message. J. Climate, 22, 6501-6514, https:// doi.org/10.1175/2009JCLI3062.1.
Norquist, D. C., E. E. Recker, and R. J. Reed, 1977: The energetics of African wave disturbances as observed during phase III of GATE. Mon. Wea. Rev., 105, 334-342, https://doi.org/10.1175/ 1520-0493(1977)105<0334:TEOAWD>2.0.CO;2.

North, G. R., T. L. Bell, R. F. Cahalan, and F. J. Moeng, 1982: Sampling errors in the estimation of empirical orthogonal functions. Mon. Wea. Rev., 110, 699-706, https://doi.org/ 10.1175/1520-0493(1982)110<0699:SEITEO > 2.0.CO;2.

Philippon, N., F. J. Doblas-Reyes, and P. M. Ruti, 2010: Skill, reproducibility and potential predictability of the West African monsoon in coupled GCMs. Climate Dyn., 35, 53-74, https:// doi.org/10.1007/s00382-010-0856-5.

Pytharoulis, I., and C. Thorncroft, 1999: The low-level structure of African easterly waves in 1995. Mon. Wea. Rev., 127, 22662280, https://doi.org/10.1175/1520-0493(1999)127<2266: TLLSOA $>2.0 . \mathrm{CO} ; 2$.

Reed, R. J., D. C. Norquist, and E. E. Recker, 1977: The structure and properties of African wave disturbances as observed during phase III of GATE. Mon. Wea. Rev., 105, 317-333, https://doi.org/ 10.1175/1520-0493(1977)105<0317:TSAPOA > 2.0.CO;2.

_ E. Klinker, and A. Hollingsworth, 1988: The structure and characteristics of African easterly wave disturbances as determined from the ECMWF operational analysis/forecast system. Meteor. Atmos. Phys., 38, 22-33, https://doi.org/ 10.1007/BF01029944.

Ruti, P. M., and A. Dell'Aquila, 2010: The twentieth century African easterly waves in reanalysis systems and IPCC simulations, from intra-seasonal to inter-annual variability. Climate Dyn., 35, 1099-1117, https://doi.org/10.1007/s00382-010-0894-z.

Rydbeck, A. V., and E. D. Maloney, 2014: Energetics of east Pacific easterly waves during intraseasonal events. J. Climate, 27, 7603-7621, https://doi.org/10.1175/JCLI-D-14-00211.1.

Schlueter, A., A. H. Fink, P. Knippertz, and P. Vogel, 2019a: A systematic comparison of tropical waves over northern Africa. Part I: Influence on rainfall. J. Climate, 32, 1501-1523, https:// doi.org/10.1175/JCLI-D-18-0173.1.

$\longrightarrow, \ldots$, and — 2019b: A systematic comparison of tropical waves over northern Africa. Part II: Dynamics and thermodynamics. J. Climate, 32, 2605-2625, https://doi.org/10.1175/ JCLI-D-18-0651.1.

Sultan, B., S. Janicot, and A. Diedhiou, 2003: The West African monsoon dynamics. Part I: Documentation of intraseasonal variability. J. Climate, 16, 3389-3406, https://doi.org/10.1175/ 1520-0442(2003)016<3389:TWAMDP >2.0.CO;2.

Thorncroft, C. D., and B. J. Hoskins, 1994: An idealized study of African easterly waves. I: A linear view. Quart. J. Roy. Meteor. Soc., 120, 953-982, https://doi.org/10.1002/qj.49712051809.

_ N. M. J. Hall, and G. N. Kiladis, 2008: Three-dimensional structure and dynamics of African easterly waves. Part III: Genesis. J. Atmos. Sci., 65, 3596-3607, https://doi.org/10.1175/ 2008JAS2575.1.

— H. Hguyen, C. Zhang, and P. Peyrillé, 2011: Annual cycle of the West African monsoon: Regional circulations and associated water vapour transport. Quart. J. Roy. Meteor. Soc., 137, 129-147, https://doi.org/10.1002/qj.728.

Yang, G.-Y., J. Methven, S. Woolnough, K. Hodges, and B. Hoskins, 2018: Linking African easterly wave activity with equatorial waves and the influence of Rossby waves from the Southern Hemisphere. J. Atmos. Sci., 75, 1783-1809, https:// doi.org/10.1175/JAS-D-17-0184.1.

Zawislak, J., and E. J. Zipser, 2010: Observations of seven African easterly waves in the east Atlantic during 2006. J. Atmos. Sci., 67, 26-43, https://doi.org/10.1175/2009JAS3118.1. 